\title{
Artigo \\ Antena de microfita com sobrecamada de cera de carnaúba
}

\author{
Stefany Kariny dos Santos de Souza Queiroz ${ }^{[1]}$, Idalmir de Souza Queiroz Júnior ${ }^{\text {[2] }}$ \\ [1] Universidade Federal Rural do Semi-árido; stefanykariny94@gmail.com \\ [2] Universidade Federal Rural do Semi-árido; idalmir@ufersa.edu.br \\ Recebido: 26/06/2019; \\ Aceito: 21/08/2019; \\ Publicado: 07/10/2019.
}

Resumo: O crescente desenvolvimento das telecomunicações, particularmente a de dispositivos móveis, têm estimulado as pesquisas relacionadas as antenas de microfita nos últimos anos, visto que são os elementos fundamentais na cadeia de transmissão e recepção de sinais. Estas antenas são vantajosas por serem discretas, moldáveis a superfícies variadas, simples de serem construídas, de baixo custo, mecanicamente compatíveis com projetos de circuito integrado monolítico de micro-ondas (MMIC), além da versatilidade de projeto. Considerando a abundância da cera de carnaúba, que é proveniente de uma espécie de palmácea, e por possuir relevância social, econômica e cultural no cenário do semiárido nordestino, este trabalho aborda a aplicação da cera de carnaúba como sobrecamada (ou superestrato) para antenas retangulares de microfita. O interesse do trabalho é compreender o comportamento eletromagnético da multicamada em antenas de microfita. Neste trabalho, inicialmente foi projetada uma antena de microfita retangular padrão pelo método de linha de transmissão e o método da cavidade, após isso a antena foi simulada no Ansoft HFSS (High Frequency Structural Simulator) duas antenas: a antena padrão projetada (sem sobrecamada) e a antena projetada com sobrecamada de cera de carnaúba, para o estudo de interesse. Com a simulação foi possível obter os diagramas de radiação (2D e 3D), perda de retorno, carta de Smith e ganho para ambas as antenas, para que fosse possível compará-las, e por fim, foram confeccionadas para que se pudesse testá-las para poder validar os dados do projeto e das simulações. Os resultados simulados nas antenas foram realizados para ressoar na frequência de $2,45 \mathrm{GHz}$. Observou-se que houve uma boa concordância dos resultados simulados e testados, observados através do diagrama perda de retorno e da carta de Smith, além de um significativo aumento na perda de retorno da antena com a sobrecamada de cera quando comparada a antena padrão.

Palavras-chave: antena de microfita; cera de carnaúba; micro-ondas

\section{INTRODUÇÃO}

A área de Telecomunicações no Brasil e no mundo tem mudado a maneira de viver, produzir e de se relacionar da população nas últimas duas décadas. A crescente evolução nos sistemas de informação acabou fazendo com que mais investimentos fossem realizados no setor das Telecomunicações. Segundo a Associação Brasileira de Telecomunicação [1], a receita bruta deste setor foi de aproximadamente $\mathrm{R} \$ 240,0$ bilhões em 2014, foi o valor mais alto da história do setor de telecomunicações e equivaleu a 4,2\% do PIB (Produto Interno Bruto). Já em 2018, ainda segundo a Associação Brasileira de Telecomunicações, nos noves primeiros meses de 2018 as prestadoras de serviços de telecomunicações investiram R \$ 20,5 bilhões na expansão, modernização e melhoria da qualidade de serviços. Os investimentos corresponderam a $2,6 \%$ da Formação Bruta do Capital Fixo e a 0,41\% do PIB no período. Em decorrência desse progresso é necessário que cada vez mais esse setor se desenvolva e receba investimentos.

As antenas de microfita, a princípio, foram propostas por G. A. Deschamps no ano de 1953, mas tornaramse populares na década de 70, quando um grupo de pesquisadores incluindo Robert E. Munsonas desenvolveram quando utilizaram um substrato de baixa perda [2]. Consiste basicamente em duas placas condutoras, que são separadas por um material dielétrico, o substrato. São bastante utilizadas em aparelhos portáteis e móveis. Sua 
utilização não é uma novidade no mundo das comunicações. Atualmente, desempenham um papel importante nas telecomunicações, sendo disponíveis em diversas configurações. Recentemente, novos materiais vêm sendo utilizados na confecção de antenas, com intuito de melhorar algum parâmetro, seja através de dopagem, de misturas de compósitos ou uso de multicamadas. Esses novos materiais apresentam uma promissora área de pesquisa que promete trazer importantes avanços científicos em várias áreas, como nas telecomunicações, microeletrônica e até mesmo área médica.

Neste trabalho, será utilizada a cera de carnaúba, que é um material regional com grande valor cultural, social e econômico. Pretende-se estudar o comportamento desse material quando utilizado sobre uma antena de microfita.

\section{MATERIAIS E MÉTODOS}

Atualmente, as antenas são estruturas importantes nas comunicações e para o desenvolvimento tecnológico humano. Segundo Balanis [3], uma antena é a estrutura intermediária entre o espaço livre e a linha de transmissão, que é utilizada para transportar a energia eletromagnética. De forma simplificada, o mecanismo de radiação de uma antena pode ser entendido como uma fonte que gera campos eletromagnéticos que são confinados e guiados pela linha de transmissão e antena, e por fim, quando essa perturbação se aproxima da abertura da antena é formada uma onda no espaço livre.

$\mathrm{Na}$ Figura 1 pode se observar uma antena de Microfita. Esta antena pertence a uma classe de antenas, chamada de antena planar. Usadas em diversas aplicações onde o perfil fino e o baixo peso são importantes.

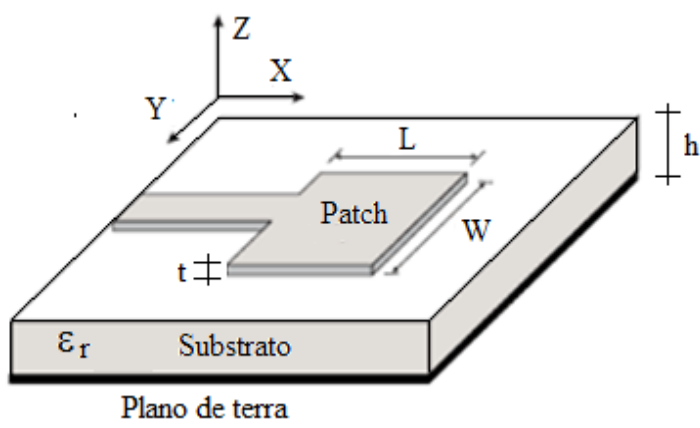

FIGURA 1. Antena de microfita, onde L representa a largura do patch (elemento radiador), W representa o comprimento do patch, $\mathrm{t}$ representa a espessura do patch, $\mathrm{h}$ representa a altura do substrato e $\varepsilon_{\mathrm{r}}$ representa a permissividade elétrica relativa do material do substrato [3].

De forma simplificada, a antena de microfita consiste em uma placa metálica, comumente conhecida como patch (elemento radiador), colocada sobre um substrato (dielétrico) que por sua vez está sobre um plano de terra, uma camada metálica fina. O patch pode ter diversas geometrias, pode ser: quadrado, retangular, circular, elíptico, triangular, etc. O material e forma do patch são elementos que influenciam diretamente no desempenho da antena. Geralmente, o patch é fabricado de cobre ou ouro [4].

O Modelo da linha de transmissão será o método utilizado neste trabalho para projetar a antena. Este método leva em consideração os efeitos de borda causados pelo fato do patch possuir dimensões finitas, o que leva a um efeito de franjeamento, onde a quantidade será função das dimensões do patch e do substrato. Segundo o Balanis [3], para determinar os parâmetros necessários para a antena de microfita através do método da linha de transmissão, é necessário calcular: a largura do elemento, o verdadeiro comprimento, o comprimento efetivo e a frequência de ressonância, que são dadas pelas seguintes equações:

a) A largura $\mathrm{W}$ do elemento radiador é dada pela Equação 1

$$
\mathrm{W}=\frac{1}{2 \mathrm{f}_{\mathrm{r}} \sqrt{\mu_{0} \varepsilon_{0}}} \sqrt{\frac{2}{\varepsilon_{\mathrm{r}}+1}}=\frac{\mathrm{v}_{0}}{2 \mathrm{f}_{\mathrm{r}}} \sqrt{\frac{2}{\varepsilon_{\mathrm{r}}+1}}
$$

Sendo a constante dielétrica efetiva da antena determinada pela Equação 2.

$$
\varepsilon_{\mathrm{ref}}=\frac{\varepsilon_{\mathrm{r}}+1}{2}+\frac{\varepsilon_{\mathrm{r}}-1}{2}\left(1+12 \frac{\mathrm{h}}{\mathrm{W}}\right)^{-1 / 2}
$$


b) Após determinar o valor de W, determina-se a partir da Equação 3 a extensão de comprimento gerada pelos campos de bordas.

$$
\frac{\Delta \mathrm{L}}{\mathrm{h}}=0,412 \frac{\left(\varepsilon_{\mathrm{ref}}+0,3\right)\left(\frac{\mathrm{W}}{\mathrm{h}}+0,264\right)}{\left(\varepsilon_{\mathrm{ref}}-0,258\right)\left(\frac{\mathrm{W}}{\mathrm{h}}+0,8\right)}
$$

c) O comprimento verdadeiro da antena é dado pela Equação 4.

$$
\mathrm{L}=\frac{1}{2 \mathrm{f}_{\mathrm{r}} \sqrt{\varepsilon_{\text {ref }}} \sqrt{\mu_{0} \varepsilon_{0}}}-2 \Delta \mathrm{L}
$$

d) O comprimento efetivo da antena é calculado pela Equação 5.

$$
\mathrm{L}_{\mathrm{ef}}=\mathrm{L}+2 \Delta \mathrm{L}
$$

e) A frequência de ressonância é expressa pela Equação 6.

$$
\left(\mathrm{f}_{\mathrm{r}}\right)_{010}=\frac{\mathrm{v}_{0}}{2 \mathrm{~L} \sqrt{\varepsilon_{\mathrm{r}}}}
$$

Onde temos que:

$\mathrm{W}$ - largura do patch;

$\mathrm{L}$ - comprimento do patch;

$\Delta \mathrm{L}$ - variação de comprimento da antena;

$\mathrm{f}_{\mathrm{r}}$ - frequência de ressonância;

$\varepsilon_{\text {ref }}$ - constante elétrica efetiva;

$\mathrm{v}_{\mathrm{o}}$ - velocidade das ondas eletromagnéticas no espaço livre;

$\varepsilon_{\mathrm{r}}$ - constante elétrica relativa do dielétrico;

$\mathrm{h}$ - espessura do substrato dielétrico.

Para realizar o casamento de impedância entre a antena e a linha de alimentação é usado um inset feed, onde os parâmetros $\mathrm{y}_{0}$ e $\mathrm{y}_{\mathrm{w}}$ precisam ser calculados. Onde $\mathrm{y}_{0}$, comprimento do inset feed, pode ser encontrado utilizando a Equação 7 e $\mathrm{y}_{\mathrm{w}}$, largura do inset feed, pode ser considerado com a mesma largura da linha de transmissão, segundo alguns autores [5]. Na Figura 2 pode ser observado o inset feed na antena.

$$
\mathrm{y}_{0}=10^{-4}\left(0,001699 \varepsilon_{\mathrm{r}}^{7}+0,13761 \varepsilon_{\mathrm{r}}^{6}-6,1783 \varepsilon_{\mathrm{r}}^{5}+93,187 \varepsilon_{\mathrm{r}}^{4}-682,69 \varepsilon_{\mathrm{r}}^{3}+2561,9 \varepsilon_{\mathrm{r}}^{2}-4043 \varepsilon_{\mathrm{r}}+6697\right) \frac{\mathrm{L}}{2}
$$

Para a linha de transmissão, é necessário calcular a largura $\mathrm{W}_{0}$, que pode ser encontrada pela Equação 8, fazendo-se a impedância da linha de microfita, $\mathrm{Z}_{0}$, igual a $50 \Omega$. O comprimento da linha de alimentação é igual a um quarto do comprimento de onda $(1 / 4 \lambda)$. A variável h representa a espessura do substrato dielétrico.

$$
\mathrm{Z}_{0}= \begin{cases}\frac{60}{\sqrt{\varepsilon_{\mathrm{ref}}}} \ln \left(\frac{8 \mathrm{~h}}{\mathrm{~W}_{0}}+\frac{\mathrm{W}_{0}}{4 \mathrm{~h}}\right), & \frac{\mathrm{W}_{0}}{\mathrm{~h}} \leq 1 \\ \frac{120 \pi}{\sqrt{\varepsilon_{\text {ref }}}\left[\frac{\mathrm{W}_{0}}{\mathrm{~h}}+1,393+0,667 \ln \left(\frac{\mathrm{W}_{0}}{\mathrm{~h}}+1,444\right)\right]}, & \frac{\mathrm{W}_{0}}{\mathrm{~h}}>1\end{cases}
$$

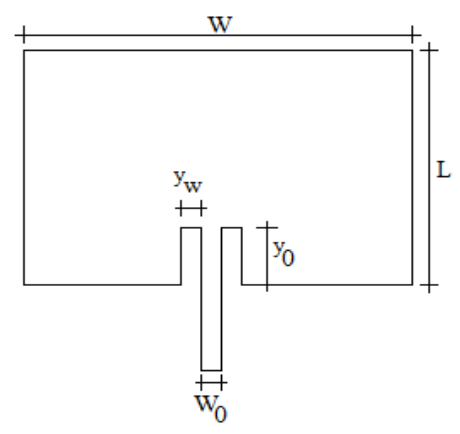

FiguRA 2. Antena de microfita e dimensões de projeto (Adaptada de [6]). 
O substrato pode ser fabricado de diversos materiais, um dos mais comuns é o FR4. Este material é um compósito de fibra de vidro com resina epóxi, sendo um termoplástico bastante versátil e mais comumente utilizado como isolante elétrico.

A cera de carnaúba é um produto natural que é extraído de uma palmeira. Possui aplicabilidade em diversos processos de industrialização de produtos que trazem benefícios a sociedade [7]. Segundo o IBGE [8], os principais estados produtores são: Piauí, Ceará, Rio Grande do Norte e o Maranhão. A cera da carnaúba é o principal produto da carnaubeira, sendo utilizada em diversos produtos no dia-a-dia, como por exemplo: batom, verniz, tintas térmicas etc. A cera da carnaúba é obtida a partir das folhas da planta, uma vez que as folhas são revestidas por uma cobertura cerífera [9]. Mais especificamente, a cera é obtida do pó da palha da carnaúba, podendo ser produzida de forma industrial ou artesanal. Após a palha ser cortada, ocorre a secagem ao sol da palha e então se extrai o pó cerífero. Após a obtenção do pó, ocorre a produção da cera bruta, onde a água é utilizada como solvente, com isso, clareia-se o pó com peróxido de hidrogênio. Segundo Cavalcante [10], o pó possui colorações diferentes, quando extraído da extensão das folhas possui cor amarela alaranjada ou preta, já quando é extraída da parte central das folhas, tem a cor amarelo ouro.

Segundo o estudo desenvolvido por Callinan e Parks [11], em temperaturas muito baixas a constante dielétrica da cera de carnaúba também é baixa, entre 2,16 a 2,29 e difere ligeiramente em diferentes frequências. A medida que a temperatura vai aumentando a constante dielétrica também aumenta, porém, inicialmente aumenta lentamente e depois de maneira mais rápida até que a constante atinja seu ponto máximo e comece a diminuir, mesmo que se continua a aumentar sua temperatura. Já para o fator de perda dielétrica, seus pontos de máximo são distintos em diferentes temperaturas, pois dependem da frequência. Em baixas frequências se obtém valores de máximo maiores, enquanto em frequências mais altas se atinge o valor de máximo e depois tende a cair. Na Figura 3 pode ser observado o comportamento da constante dielétrica e da constante de perda levando em consideração a frequência e a temperatura em uma faixa de frequência que varia de $0,1 \mathrm{kHz}$ a 100 $\mathrm{kHz}$.

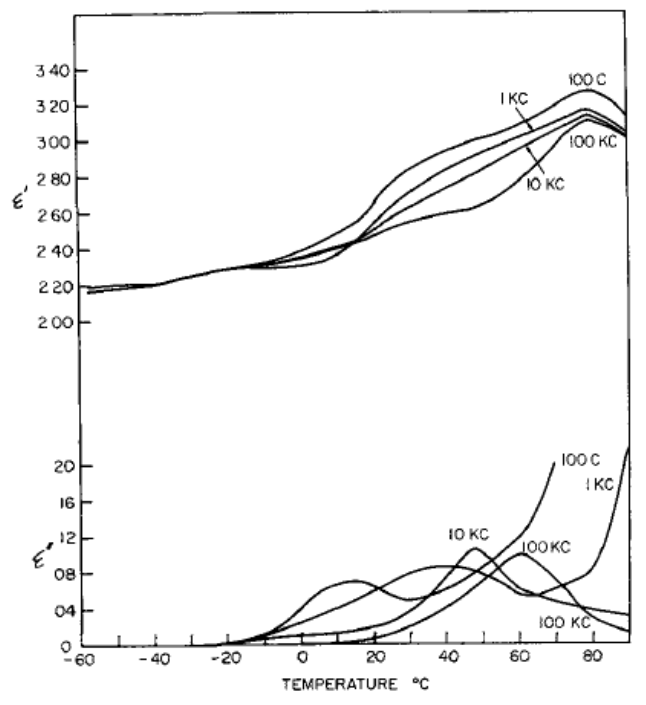

FIGURA 3. Variação da constante dielétrica e fator de perda dielétrica para a cera de carnaúba [11].

A tangente de perdas, segundo Pozar [12], pode ser definida como a relação entre a parte imaginária efetiva da permissividade e a sua parte real. De forma geral, espera-se que a tangente de perdas seja baixa na frequência de operação do dispositivo, isso faz com que ocorra uma baixa perda por inserção. Segundo Cavalcante [10], a cera da carnaúba caracterizada em seu trabalho apresentou uma permissividade elétrica relativa aproximadamente igual a 2,35 e tangente de perdas aproximadamente igual a 0,0714, para uma frequência de 2,45 GHz.

Considerando o uso de uma placa de FR4 para substrato da antena, tem-se que a altura da antena é h=1,58 $\mathrm{mm}, \varepsilon_{\mathrm{r}}=4,40$, a frequência de ressonância de projeto é $2,45 \mathrm{GHz}$ e o modo de ressonância de TM010. Usando as equações apresentadas anteriormente, foram calculados os parâmetros da antena, apresentados na Tabela 1. 
TABELA 1. Dimensões projetadas da Antena de microfita sobre substrato de FR4 (Autoria própria).

\begin{tabular}{ccccccc}
\hline \multicolumn{2}{c}{ Patch } & $\begin{array}{c}\text { Linha de } \\
\text { Transmissão }\end{array}$ & \multicolumn{2}{c}{ Inset Feed } & Substrato de FR4 & $\begin{array}{c}\text { Sobrecamada de } \\
\text { cera de carnaúba }\end{array}$ \\
\hline $\mathrm{W}(\mathrm{mm})$ & $\mathrm{L}(\mathrm{mm})$ & $\mathrm{W}_{0}(\mathrm{~mm})$ & $\mathrm{y}_{0}(\mathrm{~mm})$ & $\mathrm{y}_{\mathrm{w}}(\mathrm{mm})$ & $\mathrm{h}(\mathrm{mm})$ & $\mathrm{d}(\mathrm{mm})$ \\
\hline 37,243 & 38,824 & 3,318 & 8,853 & 3,318 & 1,58 & $1,5 \mathrm{~mm}($ aprox...) \\
\hline
\end{tabular}

As antenas projetadas com e sem sobrecamada de cera de carnaúba foram simuladas no software Ansys HFSS ${ }^{\circ}$ (High Frequency Structural Simulator) para a obtenção da perda de retorno, ganho e a carta de Smith. Para a simulação, foi criado no HFSS um novo material, a cera de carnaúba, com as propriedades elétricas encontradas na caracterização realizada por Cavalcante [10]. Na construção da antena foi usado um conector SMA-KE fêmea reverso de cobre banhado a ouro com impedância de $50 \Omega$ e faixa de operação de 0 a $6 \mathrm{GHz}$. $\mathrm{O}$ conector foi soldado à linha de transmissão e ao plano de terra, para permitir a alimentação e os testes na antena.

A sobrecamada da antena foi construída a partir da cera de carnaúba. Para a produção da sobrecamada foi utilizado um agitador magnético com aquecimento. Com isso, o pó da cera de carnaúba foi aquecido até seu ponto de fusão, que é de aproximadamente $74^{\circ} \mathrm{C}$ e mediu-se essa temperatura com o auxílio de um termostato. Após seu aquecimento, o pó derretido foi deposito em cima da antena de microfita e por fim, resfriado até a temperatura ambiente em uma estufa, para que o resfriamento ocorresse de forma lenta e gradativa evitando possíveis rachaduras na sobrecamada de cera.

Após a etapa de confecção a antena padrão e antena com a sobrecamada foram testadas com um mini VNA Tiny $-1 \mathrm{MHz}$ a $3 \mathrm{GHz}$, que é um analisador de redes vetoriais utilizado para medir a perda de retorno e impedância de entrada das antenas. Na Figura 4 é apresentada as antenas construídas e sua conexão ao mini VNA.

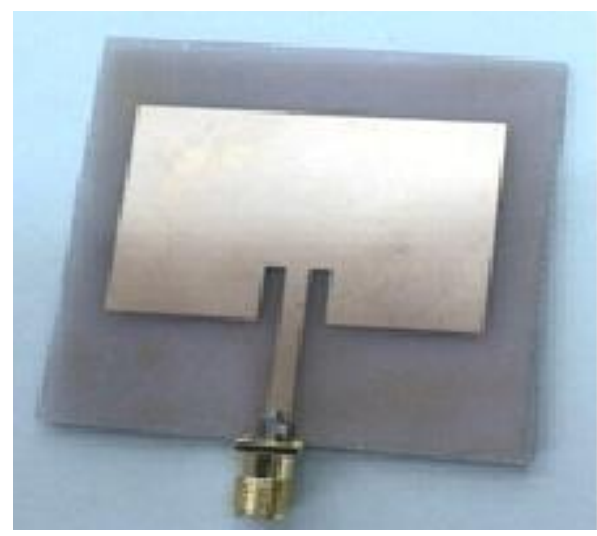

(a)

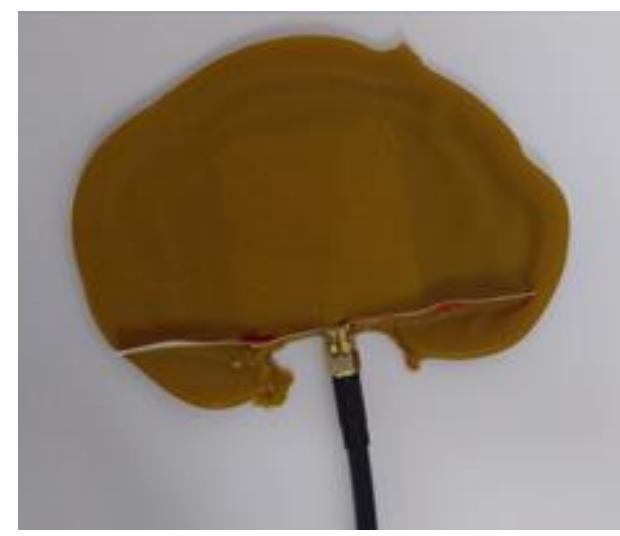

(b)

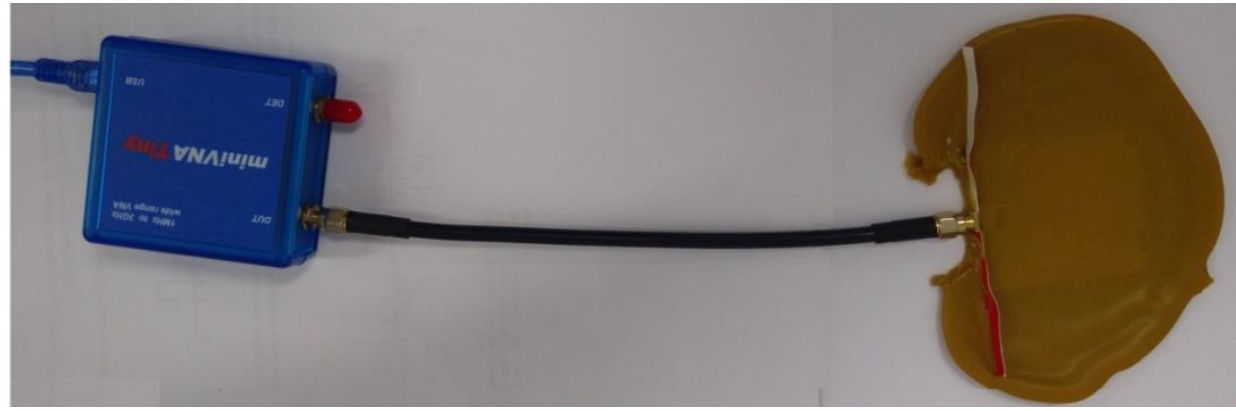

(c)

FIGURA 4. Antena de microfita com substrato de FR4. (a) Sem sobrecamada; (b) com sobrecamada; (c) antena de microfita com substrato de FR4 com sobrecamada conectada ao mini VNA (Autoria própria). 
$\mathrm{Na}$ antena fabricada pode-se perceber claramente a sobrecamada depositada sobre a antena de microfita padrão sobre substrato de FR4. A conexão da antena ao mini VNA foi simples e permitiu a medição de parâmetros importantes para comparação com resultados simulados e verificação do funcionamento da antena, apresentados na seção seguinte.

\section{RESULTADOS E DISCUSSÕES}

A partir das simulações foi possível obter dados para a construção dos resultados da perda de retorno, de $\mathrm{S}_{1,1}(\mathrm{~dB})$, das duas antenas. Na Figura 5 é mostrado os resultados da simulação da antena de microfita padrão e da antena de microfita com a sobrecamada de cera de carnaúba, a fim de que se possa comparar os dois resultados obtidos. Nesta Figura se observa a perda de retorno da antena padrão com os símbolos em forma de losango, e a perda de retorno da antena com sobrecamada com os símbolos quadrados. A linha de -10dB indica a faixa de frequência de operação, ou seja, a largura de banda de cada antena, e o ponto mais baixo da curva indica a frequência de ressonância da antena simulada.

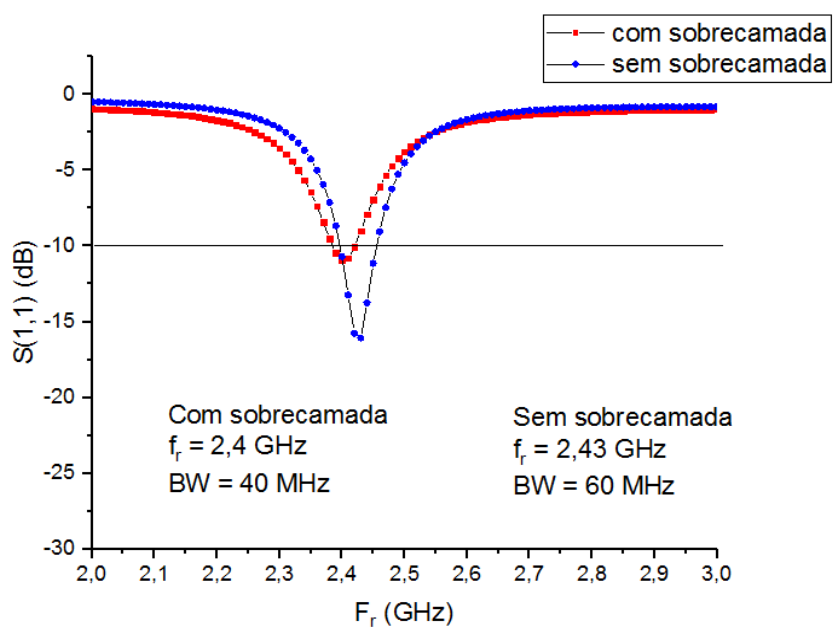

FiguRA 5. Simulação de $\mathrm{S}(1,1)(\mathrm{dB})$ da antena de microfita padrão e com sobrecamada de cera de carnaúba (Autoria própria).

Na Figura 5 percebe-se que ambas as antenas ressoam próximo da frequência de projeto. A frequência de ressonância da antena padrão é de $2,43 \mathrm{GHz}$, com largura de banda de $60 \mathrm{MHz}$, enquanto que a frequência de ressonância com sobrecamada caiu para $2,4 \mathrm{GHz}$ com largura de banda de $40 \mathrm{MHz}$. São apresentadas as simulações dos diagramas de radiação 2D das antenas sem sobrecamada e com sobrecamada na Figura 6. Percebe-se que a sobrecamada não teve muita influência sobre o diagrama de radiação com mudanças muito sutis entre as duas Figuras, quando se compara estas duas antenas. Ambas possuem aproximadamente a mesma intensidade de radiação à $0^{\circ}$, dificultando a diferenciação visual. Isso pode ser pela espessura da sobrecamada, ou pela baixa permissividade elétrica relativa da cera de carnaúba. O mesmo ocorre na Figura 7 para ao diagrama de radiação $3 \mathrm{D}$.

Na Figura 8 são apresentadas as medidas das perdas de retorno para a antena de microfita sem sobrecamada e com sobrecamada de cera de carnaúba. Observa-se que a cera de carnaúba, desloca a frequência um valor menor que a antena padrão, aumenta a largura de banda e melhora o casamento de impedância. A antena padrão ressoa em 2,4GHz, com largura de banda de 52,3MHz, próxima da simulação, enquanto que a antena com sobrecamada ressoa em $2,37 \mathrm{GHz}$ com largura de banda de $80,2 \mathrm{MHz}$.

Comparando os resultados obtidos, percebe-se que houve uma melhora significativa na perda de retorno quando se acrescentou à antena a camada de cera de carnaúba, consequentemente, também ocorreu um aumento na perda de retorno da antena com a sobrecamada. Também pode ser observado que ocorreu um pequeno deslocamento frequência de ressonância e aumento na largura de banda.

É apresentada na Figura 9, a medida impedância de entrada da antena em um sistema de eixos retangulares ortogonais e na carta de Smith, para as frequências de ressonância de cada antena, sem a sobrecamada e com a sobrecamada. Percebe-se que a antena com sobrecamada possui um melhor casamento de impedância, mostrando que além de proteger o cobre da antena de microfita retangular, a sobre camada realiza uma melhoria 
no casamento de impedância. Nesta Figura, é apresentada a impedância para a frequência de ressonância de cada antena indicada em uma linha vertical tracejada, onde se vê claramente que a parte imaginária das impedâncias é menor na antena com sobrecamada, enquanto que a parte real é mais próxima de $50 \square$ para a antena com sobrecamada.

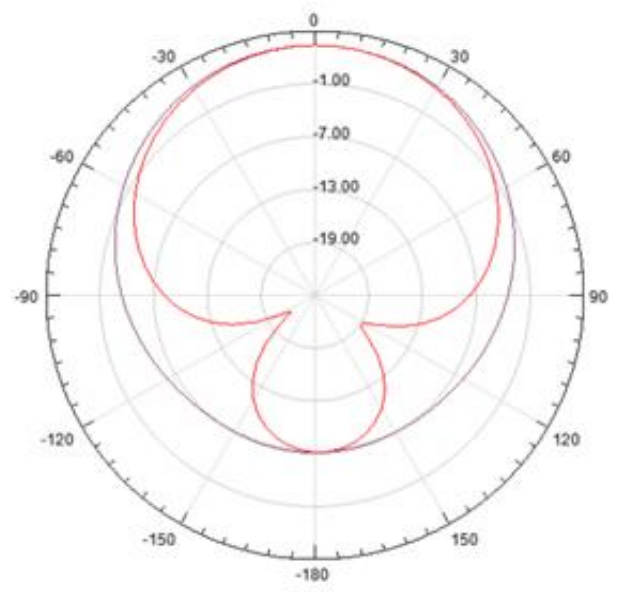

(a)

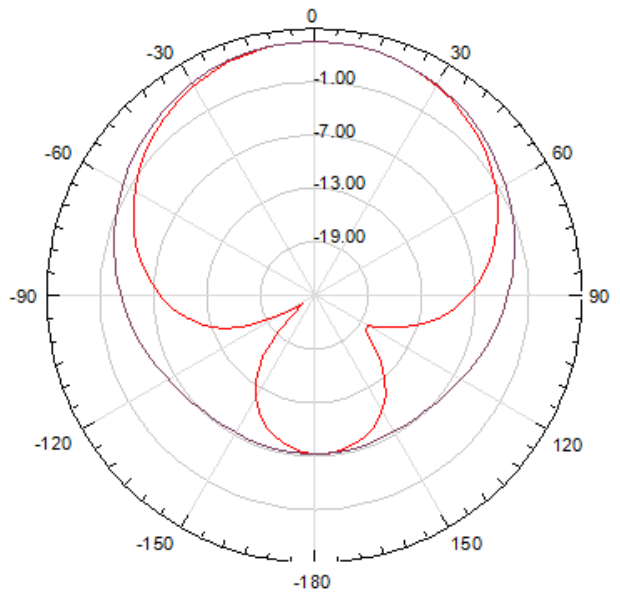

(b)

FiguRA 6. Simulação do diagrama de radiação 2D da (a) antena de microfita padrão e da (b) antena de microfita com sobrecamada (Autoria própria).
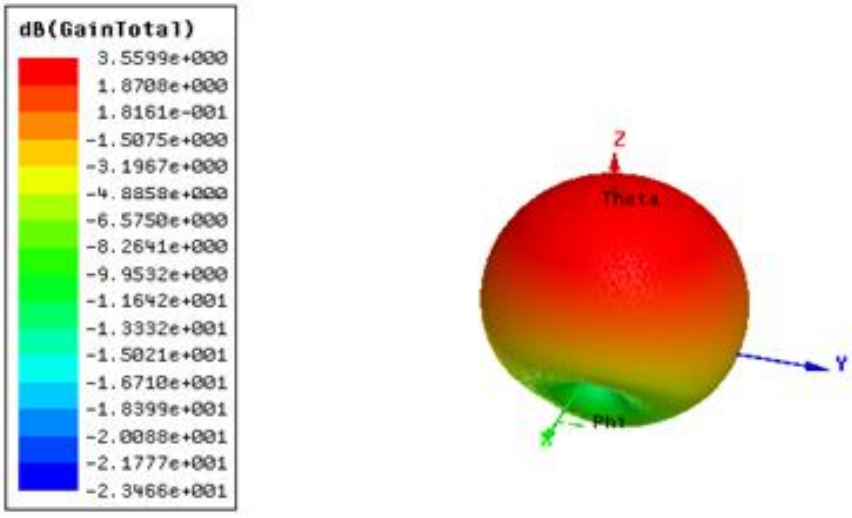

(a)
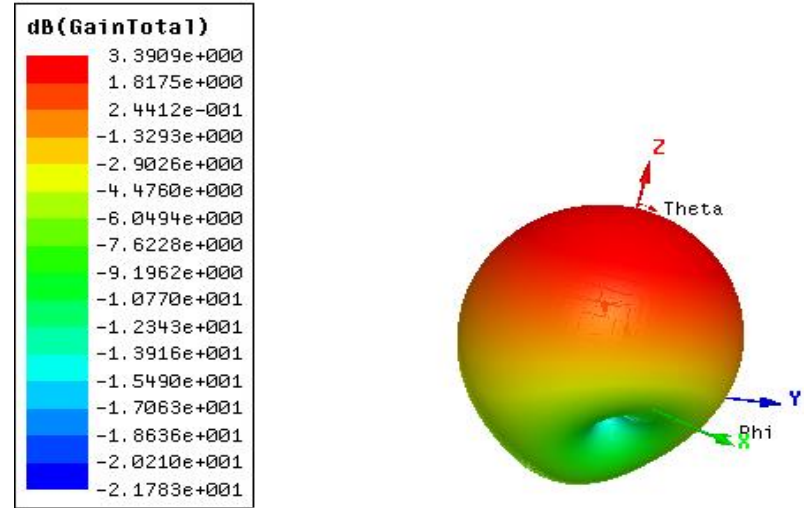

(b)

FIGURA 7. Simulação do diagrama de radiação 3D da (a) antena de microfita padrão, e da (b) antena de microfita com sobrecamada (Autoria própria). 


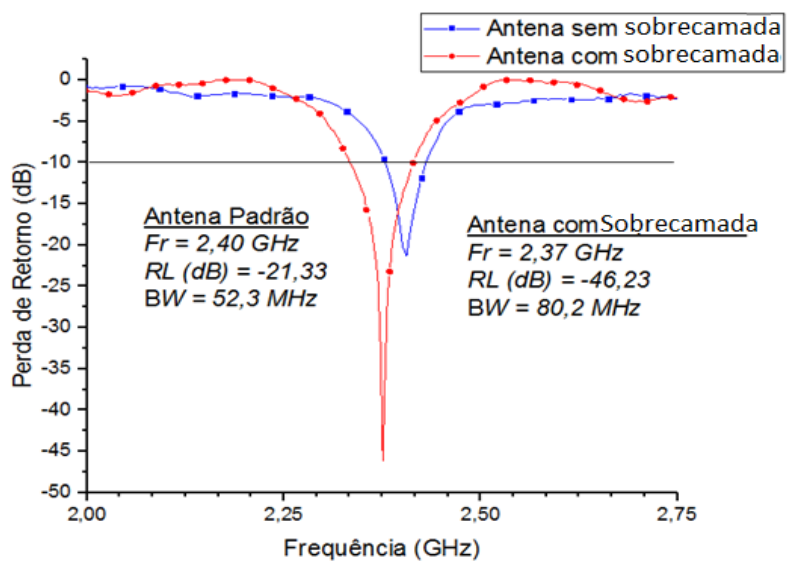

FIGURA 8. Perda de retorno medida para a antena de microfita retangular antena padrão (sem sobrecamada) e com sobrecamada (Autoria própria).

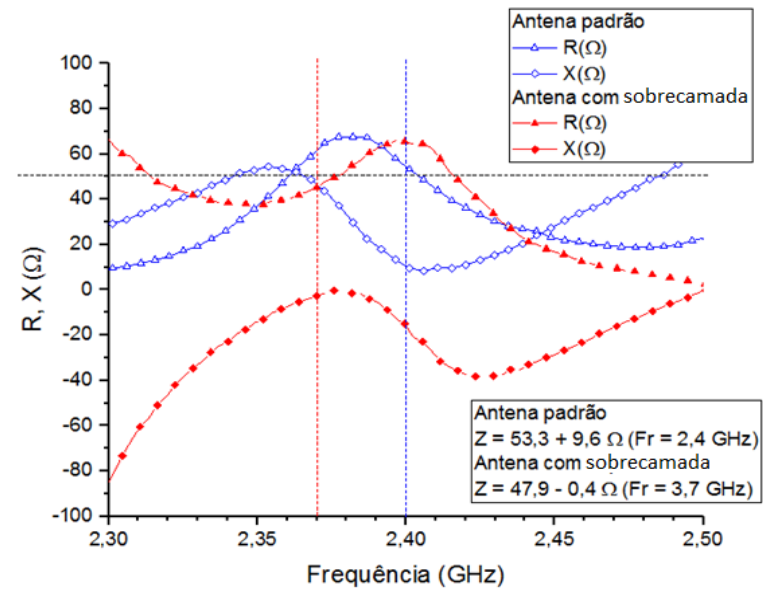

FIGURA 9. Medida linear da impedância da Antena de microfita sobre substrato de FR4 sem sobrecamada e com sobrecamada de cera de carnaúba (Autoria própria).

O mesmo efeito é observado na Figura 10, na carta de Smith, medida para as duas antenas. A impedância da antena com sobrecamada, indicada na Figura por um círculo, é mais próxima do ponto central do que a impedância da antena padrão, indicada por um quadrado. Isso indica que o casamento de impedância é melhor na antena com sobrecamada.

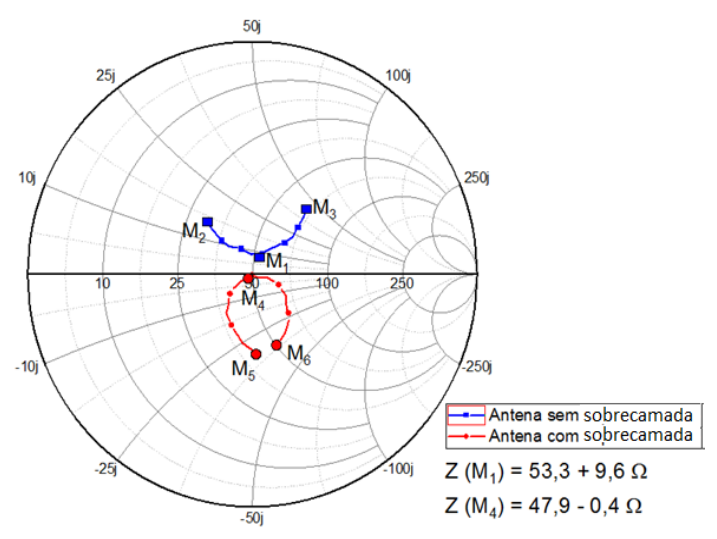

FIgURA 10. Medida da impedância da Antena de microfita sobre substrato de FR4 sem sobrecamada e com sobrecamada de cera de carnaúba na carta de Smith (Autoria própria). 
Conforme observado nas Figuras 9 e 10, a sobrecamada não serve apenas como proteção contra oxidação do patch metálico e proteção mecânica, mas também para melhora o casamento de impedância, aumentar a largura de banda e reduzir as dimensões da antena, observado a partir da redução da frequência de ressonância.

\section{CONCLUSÃO}

Com o objetivo de aplicar a cera de carnaúba aos modernos sistemas de comunicação na banda $\mathrm{S}$ (faixa de frequência de $2 \mathrm{GHz}$ e $4 \mathrm{GHz}$ ), foi realizado um estudo com aplicação prática na construção de antena de microfita com sobrecamada. A antena de microfita com sobrecamada se mostrou simples, moldáveis, de baixo custo, mecanicamente estável, além de melhorar propriedades da antena. O estudo de novos materiais para aplicações na banda $\mathrm{S}$ é diversificado, e a cera de carnaúba se mostrou muito promissora. No decorrer do trabalho foi possível obter da literatura científica os parâmetros elétricos necessários ao projeto da antena de microfita. Porém, os parâmetros elétricos da cera de carnaúba não são conhecidos pela literatura científica para frequências na faixa de $\mathrm{GHz}$, mas sim para baixas frequências. Para tanto, usou-se o trabalho de Cavalcante [10], que não apenas caracterizou a cera de carnaúba na banda $S$, como também realizou caracterização mecânica, química e elétrica. Sendo assim possível realizar a simulação da antena com sobrecamada de cera de carnaúba.

O interesse do trabalho foi compreender o comportamento eletromagnético da multicamada de cera de carnaúba em antenas de microfita, sendo este objetivo alcançado e mostrado sua viabilidade. O uso da cera de carnaúba exige uma técnica de derretimento e resfriamento lento, sendo este um pronto crítico, talvez a parte mais difícil deste trabalho. Foi projetada a antena de microfita retangular padrão, a mesma foi simulada, construída e medida, ficando seu comportamento próximo do simulado. Para que a antena tivesse um comportamento melhor seria necessário melhorar o casamento de impedância. $\mathrm{O}$ passo seguinte foi acrescentar uma sobrecamada de cera de carnaúba, para o estudo de interesse. Para esta antena foi realizada a simulação, construção e medida dos parâmetros da antena para que fosse possível compará-la com a antena padrão. Os resultados simulados e medidos das duas antenas foram comparados. Os resultados simulados não se mostraram tão bons, exceto pelo fato de que a frequência de ressonância foi deslocada para a esquerda, mostrando que o uso da sobrecamada permite uma redução nas dimensões da antena. A comparação dos resultados medidos se mostrou muito melhores na antena com sobrecamada, não apenas confirmou o deslocamento da frequência apresentado nas simulações, mas mostrou uma melhoria na perda de retorno, largura de banda e no casamento de impedância. Como trabalho futuro se sugere que o estudo das características da cera se aprofunde, e se faça uso de alguma técnica para aumentar a temperatura de fusão e da resistência mecânica.

\section{AgRADECIMENTOS}

Os autores agradecem à UFERSA pelo apoio, ao Prof. Me. Carlos Eduardo Chaves Cavalcante pela participação e colaboração neste trabalho.

\section{REFERÊNCIAS}

[1] TELEBRASIL - Associação Brasileira de Telecomunicações. "O Desempenho do Setor de Telecomunicações no Brasil Séries Temporais 9M18”. (2018). Disponível em: <http://www.telebrasil.org.br/panorama-do-setor/desempenho-do-setor>. Acesso em 02 de mar. de 2018.

[2] MEHTA, A. "Microstrip Antenna". International Journal of Scientific \& Technology Research, v. 4, n. 3, p. 54-57, 2015.

[3] BALANIS, C. A. "Teoria das antenas: análise e síntese”. vol. 1 e 2. $3^{\text {a }}$ ed. Rio de Janeiro: LTC, 2005.

[4] OLIVEIRA, M. A. et al. "Análise Paramétrica em uma Antena Patch Retangular de Microfita com Fendas”. INNOVER, Maranhão, v. 1, n. 4, p.48-60, dez. 2014.

[5] BASÍliO L. I., KHAYAT M A., WILliAMS J. T., LONG S. A., "The Dependence of the Input Impedance on Feed Position of Probe and Microstrip Line - Fed patch Antennas", IEEE Trans. Antennas Propagat, Vol.49, pp.45-47, Jan. 2001.

[6] SILVA, J. C. et al. “ $\neg \neg$ Análise experimental do efeito de diversas configurações de inset feed na alimentação de antenas planares dos tipos retangular, circular e triangular”. Principia, João Pessoa, v. 24, n. 4, p.49-56, jun. 2014.

[7] NASCIMENTO, E. B. et al. "A Cera de Carnaúba: Origem, Produção e Mercados”. VIII Encontro de Engenharia de Produção Agroindustrial. Universidade Estadual do Paraná. Anais. 2018. 
[8] IBGE - “Quantidade e valor dos produtos da extração vegetal, por produtos, segundo as Grandes Regiões e as Unidades da Federação - 2012”. Disponivel em: <ftp://ftp.ibge.gov.br/Producao_Agricola/Producao_da_Extracao_Vegetal_e_da_Silvicultura_[anual]/20 12/pdf/tab02_ceras.pdf>. Acesso em: jan, 2019.

[9] SBRT. "DOSSIÊ TÉCNICO: Cadeia produtiva da carnaúba". Bahia: Serviço Brasileiro de Normas Técnicas, 2013.

[10] CAVAlCANTE, C. E. C. "Caracterização de Propriedades da Cera de Carnaúba para Aplicação na Faixa de Frequência de Micro-Ondas como Substrato de Antenas de Microfita”. 2019. 100 f. Dissertação (Mestrado) - Curso de Engenharia Elétrica, Universidade Federal Rural do Semi - Árido, Mossoró, 2019.

[11] CALLINAN, T. D.; PARKS, A. M. "The Dielectric Constants and Loss Factors of Some Natural Waxes. In: Electrical Insulation”, 1959 Conference On. IEEE, 1959. p. 45-50.

[12] POZAR, D. M. “Microwave Engineering”. 2 ed. United States of America: John Wiley \& Sons. 1998. 